\title{
UTILIZAÇÃO DE DIFERENTES METODOLOGIAS PARA DETERMINAÇÃO DA ÁREA DE OLHO DE LOMBO EM OVINOS
}

\author{
USING DIFFERENT METHODOLOGIESTODETERMINATION \\ THE LOIN EYE AREA IN SHEEP
}

\author{
Costa, R.G. ${ }^{1 *}$, Lima, A.G.V.O. ${ }^{1}$, Oliveira, C.F.S. ${ }^{1}$, Azevedo, P.S. ${ }^{1}$ e Medeiros, A.N. ${ }^{1}$ \\ 1'Programa de Pós Graduação em Zootecnia. Universidade Federal da Paraíba. Areia-PB. Brasil. \\ *betogermano@hotmail.com
}

\section{Palavras ChaVE ADICIONAIS}

AutoCad. Carcaça. Carne ovina. Longissimus dorsi.

\section{RESUMO}

Foi avaliada a precisão das metodologias: Grade-UNESP, Grade, Geométrico, comparadas ao AutoCad, na determinação da área de olho de lombo-AOL de 40 ovinos inteiros Santa Inês com diferentes pesos corporais. Na meia-carcaça esquerda, foi realizado um corte transversal, à altura da $13^{\underline{a}}$ costela com a primeira vértebra lombar, para a mensuração AOL, através do traçado do contorno do músculo em folha plástica de transparência e caneta apropriada. Foi realizada uma análise quantitativa de precisão utilizando o modelo percentual do erro médio absoluto, para avaliar os desvios. A metodologia que apresentou menor desvio foi a Grade-UNESP, seguidos da Grade e o Geométrico. O método Grade-UNESP mostrou-se o mais preciso comparado ao AutoCad, economicamente viável e aplicável dentro da programação de abate, sendo o mais recomendado para carcaça ovina.

\section{SUMMARY}

The accuracy of the methodologies: UNESPGrade, Grade, Geometric, compared to AutoCad, in determining of the area rib eye-AOL was studied. Forty Santa Inês intact male lambs with different body weights were used. Left half carcasses were conducted a cross-sectional at the level of $13^{\text {th }}$ rib and the first lumbar vertebra, for measuring $\mathrm{AOL}$, by tracing the outline of muscle on plastic sheet of transparency and appropriate pen. We performed a quantitative analysis of the model accuracy using mean absolute percentage error,

\section{AdDICIONAL KEYWORDS}

AutoCad. Carcass. Longissimus dorsi. Sheep meat.

to measure the deviations. The lesser deviation was observed for Grade-UNESP, followed by the Grade and Geometric. Grade-UNESP method proved to be the more accurate compared to AutoCad, economically viable and applicable in the slaughter program, it was the most recommended for lamb carcass.

\section{INTRODUÇÃO}

Diante da preocupação com a qualidade da carcaça, principalmente para a comercialização, é de extrema importância sua conformação, pois características desejáveis como a conformação compacta, curta e larga, com pernas globosas e planos musculares desenvolvidos, com predominância de perfis convexos possibilitando maiores porcentagens de cortes nobres (pernil e palheta), maior proporção de músculo e menores proporções de gordura e osso apresentam maior valor comercial (Macedo etal., 2000).

A determinação da área de olho de lombo (AOL) é considerada um bom indicador da composição corporal, pois determina o conteúdo da carne de cada animal, obtendo importante influência na avaliação do preço final da carne e da classificação da carcaça (Cezar e Sousa, 2007). Quando analisada a AOL de um animal abatido, a mesma é esti- 
mada através da área da seção transversal do músculo Longissimus dorsi na altura da última costela.

Para escolher a metodologia apropriada, Yáñez et al. (2006), verificaram que há algumas exigências básicas, tais como a viabilidade, facilidade de aplicação principalmente para a avaliação da carcaça no abatedouro, baixo custo, velocidade de determinação e precisão, onde os sistemas computadorizados estão sendo empregados para reduzir os erros ou diferenças conferidas pelos operadores, sendo bastante importante para satisfazer as necessidades da indústria. Mas que podem variar em exatidão e precisão, de acordo com peso e condição corporal do animal abatido.

O objetivo deste estudo foi avaliar a precisão de diferentes metodologias, comparadas ao AutoCad, na determinação da AOL em ovinos inteiros Santa Inês de diferentes pesos corporais.

\section{MATERIALEMÉTODOS}

Foram utilizados 40 ovinos machos inteiros da raça Santa Inês, com peso médio inicial de $22,1 \mathrm{~kg}$, distribuídos em cinco tratamentos que constituíram em níveis crescentes de inclusão da palma forrageira na dieta $(0 ; 12,5 ; 25 ; 37,5 ; 50 \%)$. O delineamento experimental, utilizado foi o de blocos casualisados, com cinco tratamentos e oito repetições, onde os blocos foram formados em função do peso corporal inicial dos animais. Os animais foram identificados individualmente e distribuídos randomicamente em baias individuais. Foram abatidos aos 49 dias de experimento, sendo insensibilizados, por atordoamento, na região atla-occipital, seguida por sangria, através da secção da carótida e jugular. Após o abate, as carcaças foram resfriadas por 24 horas $\left( \pm 2 \mathrm{a} 4^{\circ} \mathrm{C}\right)$ em câmara frigorífica, seccionadas ao meio e na meia-carcaça esquerda foi realizado um corte transversal, à altura da $13^{\mathrm{a}}$ costela com a primeira vértebra lombar, para mensuração da área de olho

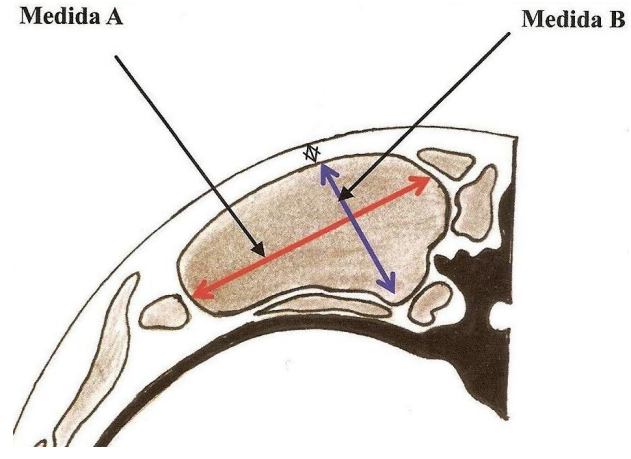

Figura 1. Método geométrico. (Geometric method).

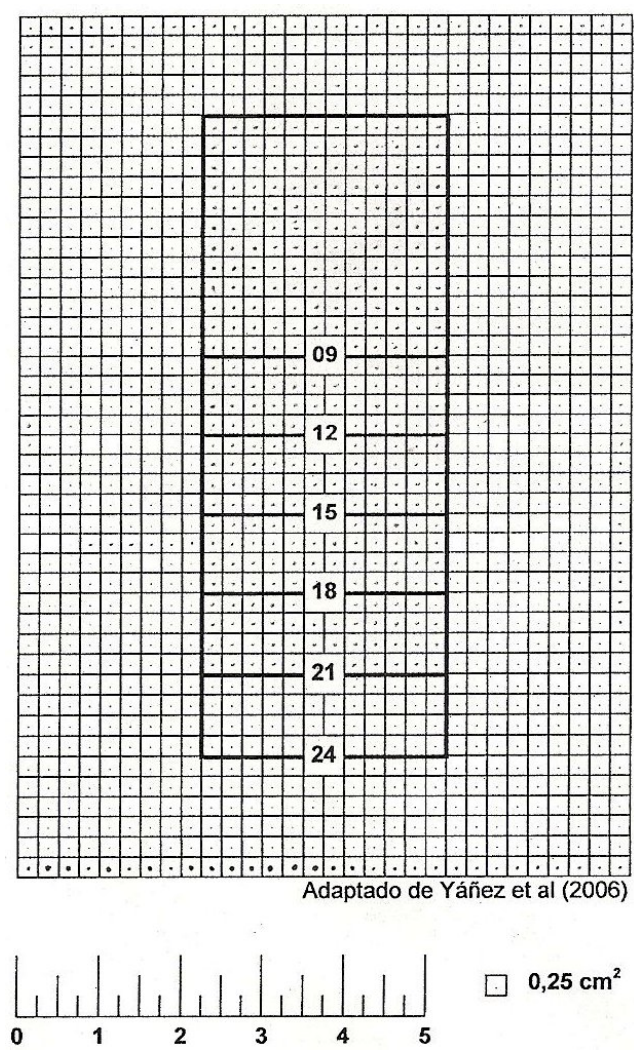

Figura 2. Método Grade-UNESP $\left(0,25 \mathrm{~cm}^{2}\right)$. (Grade UNESP method). 


\section{METODOLOGIAS PARA DETERMINAÇÃO DA ÁREA DE OLHO DE LOMBO}

de lombo (AOL) do músculo Longissimus dorsi, através do traçado do contorno do músculo em folha plástica de transparência e caneta apropriada, para determinação da área por diferentes metodologias:

a) Método geométrico: com uma régua foram traçadas duas retas, medindo a largura do olho de lombo (A), ou seja, a distância máxima do músculo a partir da linha medial à extremidade lateral dos longíssimos torácicos e lombares e à profundidade (B), distância máxima perpendicular à largura, localizada adjacente à borda lateral das vértebras. Em seguida, foi calculada a AOL a partir da equação $(\mathrm{A} / 2 \times \mathrm{B} / 2) \times \pi$, considerando $\pi=3,1416$ (figura 1 ).

b) Método Grade: consiste na utilização de uma grade plástica quadriculada, onde cada quadrado mede $10 \times 10 \mathrm{~mm}$, obtendo uma área de $1 \mathrm{~cm}^{2}$ com o um ponto no seu centro. A medida foi obtida pela adição de todos os quadrados encontrados dentro do perímetro de seguimento do olho de lombo e daqueles que no contorno do traçado passou através do ponto central e os que não passaram foram desconsiderados.

c) Método Grade-UNESP: corresponde a uma grade quadriculada, onde cada quadrado apresenta $0,25 \mathrm{~cm}^{2}$ de área com um ponto no seu centro. Ao colocar a grade sobre a imagem da AOL, a obtenção da área é realizada do mesmo modo do metodo Grade, acima descrita. Quando obtido o somatórios de quadrados, este valor deve ser multiplicado por 0,25 (Yáñez et al., 2006) (figura 2).

d) Software AutoCad: as imagens da AOL foram fotografadas em cima de um papel milimetrado, para obtenção de um ponto de referência, e transferidas para o programa AutoCad. Contornando toda a figura pela parte de dentro e utilizou-se o maior número de pontos possíveis para se obter uma maior precisão das áreas. Ao formar a figura com a ligação dos pontos, o AutoCad processa a imagem e determina a área em $\mathrm{cm}^{2}$.

Na comparação entre as metodologias avaliadas para determinação de área de olho
Tabela I. Percentual médio da área de olho de lombo $\left(\mathrm{cm}^{2}\right)$ utilizando diferentes metodologias. (Average percentage of loin eye area $\left(\mathrm{cm}^{2}\right)$ using different methodologies).

\begin{tabular}{|c|c|c|c|}
\hline \multirow{2}{*}{$\begin{array}{l}\text { AutoCad } \\
\text { (Padrão) }\end{array}$} & \multicolumn{3}{|c|}{ Metodologias } \\
\hline & Geométrico & Grade & Grade-UNESP \\
\hline 8,31 & 8,91 & 8,0 & 8,44 \\
\hline 9,98 & 11,03 & 11,0 & 10,63 \\
\hline 7,30 & 7,59 & 9,0 & 7,50 \\
\hline 7,49 & 7,31 & 9,0 & 7,88 \\
\hline 9,12 & 9,42 & 10,0 & 9,06 \\
\hline 8,02 & 9,03 & 8,0 & 8,19 \\
\hline 6,31 & 6,57 & 7,0 & 6,75 \\
\hline 6,59 & 7,85 & 7,0 & 6,88 \\
\hline 7,42 & 7,07 & 7,0 & 6,75 \\
\hline 9,31 & 10,41 & 9,0 & 9,31 \\
\hline 8,54 & 10,02 & 9,0 & 8,88 \\
\hline 7,49 & 10,01 & 9,0 & 8,00 \\
\hline 7,43 & 7,92 & 8,0 & 7,69 \\
\hline 8,82 & 9,57 & 9,0 & 9,38 \\
\hline 7,78 & 7,95 & 8,0 & 8,06 \\
\hline 8,02 & 9,42 & 9,0 & 9,00 \\
\hline 8,76 & 10,37 & 10,0 & 9,56 \\
\hline 8,30 & 8,78 & 9,0 & 8,75 \\
\hline 10,40 & 11,03 & 10,0 & 10,50 \\
\hline 9,11 & 10,01 & 11,0 & 9,63 \\
\hline 8,63 & 8,41 & 9,0 & 9,81 \\
\hline 9,29 & 8,25 & 9,0 & 9,56 \\
\hline 10,48 & 10,82 & 12,0 & 11,00 \\
\hline 10,41 & 11,03 & 11,0 & 10,94 \\
\hline 11,23 & 12,25 & 13,0 & 11,81 \\
\hline 7,63 & 7,95 & 8,0 & 8,19 \\
\hline 10,64 & 10,81 & 12,0 & 11,50 \\
\hline 10,11 & 13,21 & 11,0 & 10,63 \\
\hline 10,42 & 10,62 & 11,0 & 11,00 \\
\hline 11,57 & 12,90 & 13,0 & 11,88 \\
\hline 9,83 & 10,21 & 11,0 & 10,69 \\
\hline 10,66 & 10,60 & 13,0 & 10,69 \\
\hline 10,81 & 9,46 & 11,0 & 11,38 \\
\hline 11,08 & 11,00 & 12,0 & 11,50 \\
\hline 9,18 & 10,74 & 11,0 & 9,88 \\
\hline 8,67 & 7,76 & 10,0 & 9,00 \\
\hline 8,50 & 9,54 & 10,0 & 8,94 \\
\hline 9,67 & 11,03 & 12,0 & 11,63 \\
\hline 11,62 & 11,19 & 11,0 & 12,38 \\
\hline SAD & 23,588 & 19,800 & 9,719 \\
\hline MAPE & 6,693 & 5,676 & 2,783 \\
\hline
\end{tabular}

$\mathrm{SAD}=$ soma absoluta dos desvios $=\Sigma$ abs $\left(\mathrm{y}_{\mathrm{t}}-\hat{y}_{\mathrm{t}}\right)$. $\mathrm{MAPE}=$ percentual do erro médio absoluto. 


\section{COSTA, LIMA, OLIVEIRA, AZEVEDO E MEDEIROS}

de lombo, os dados foram compilados em planilhas EXCEL para a realização de uma análise quantitativa de precisão utilizando o AutoCad como a metodologia padrão e o modelo Percentual do Erro Médio Absoluto (MAPE), para avaliação dos desvios, metodologia adotada por Oberstone (1990).

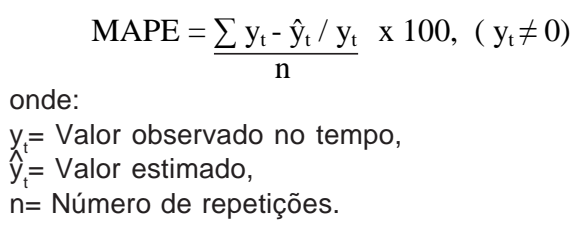

\section{RESULTADOSEDISCUSSÃO}

A metodologia que apresentou menor desvio foi a Grade-UNESP, ou seja, foi o método que mais se aproximou da metodologia padrão, seguidos da Grade e o Geométrico (tabela I).

Como a metodologia para determinação de AOL utilizando o software AutoCad é considerada a mais exata, devido a sua automatização evitando-se assim os erros obtidos com as diferenças entre operadores, considerou-se a mesma como método padrão. Porém, esta metodologia apresenta como desvantagens o seu elevado custo para aquisição do programa, obtenção de conhecimentos específicos para a sua aplicação, necessidade da área de olho de lombo ser fotocopiada e dificuldade de execução simultânea ao abate.

\section{BIBLIOGRAFIA}

Cezar, M.F. e Sousa, W.H. 2007. Carcaças ovinas e caprinas: obtenção, avaliação e classificação. Agopecuária Tropical. Uberada. 147 pp.

Macedo, F.A.F., Siqueira, E.R., Martins, E.N. e Macedo, R.M.G. 2000. Qualidade de carcaças de cordeiros Corriedale, Bergamácia $\times$ Corriedale e Hampshire Down $\times$ Corriedale, terminados em pastagem e confinamento. Rev Bras Zootecn, 29: $1520-1527$.

Oberstone, J.1990. ManagementScience-Concepts,
Com isso, durante o abate poderiam ser utilizados os métodos Grade, Grade-UNESP e geométrico, aplicado diretamente sobre a carcaça, no abatedouro, de forma prática sem prejudicar a programação do abate, reduzindo assim o tempo de obtenção das medidas e evitando possíveis erros que possam acontecer durante o traçado da área de olho de lombo. Mas, entre as três metodologias, a Grade-UNESP apresentou resultados mais próximos ao do AutoCad, indicando com isso que o fracionamento da unidade de medida reduziu a distorção dos resultados. Este fato que não é observado no método Grade, pois é um método mais utilizado em áreas de lombo bem maiores, como a dos bovinos. Resultados semelhantes foram obtidos por Yáñez et al. (2006), mantendo assim as características econômicas e práticas sem perder a sua precisão.

O método geométrico apresentou o maior desvio apesar de ser bastante utilizado na avaliação de carcaças ovinas. Segundo Siqueira e Fernandes (2000) possui como vantagens a fácil aplicação e o baixo custo, sendo necessária apenas uma régua com graduação exata.

\section{CONCLUSÃO}

O método Grade-UNESP mostrou-se o mais preciso, economicamente viável e aplicável dentro da programação do abate, sendo o mais recomendado para avaliação.

Insights, and Applications. West Publ. Co. NY. Siqueira, E.R. e Fernandes, S. 2000. Efeito do genótipo sobre as medidas objetivas e subjetivas da carcaça de cordeiros terminados em confinamento. Rev Bras Zootecn, 29: 306-311. Yáñez, E.A., Ferreira, A.C.D., Medeiros, A.N., Pereira Filho, J.M., Teixeira, I.A.M.A. and Resende, K.T. 2006. Methodologies for ribeye area determination in goats. Small Ruminant Res, 66: 197-200. 